\title{
Réconcilier, réprimer : les "années de plomb" en Italie et les transitions démocratiques dans le cône sud latino-américain
}

\section{Daniel Mouchard}

\section{(2) OpenEdition Journals}

\section{Édition électronique}

URL : http://journals.openedition.org/conflits/479

DOI : $10.4000 /$ conflits.479

ISSN : 1777-5345

Éditeur :

CCLS - Centre d'études sur les conflits lilberté et sécurité, L'Harmattan

Édition imprimée

Date de publication : 1 septembre 2000

ISBN : 2-7475-0572-3

ISSN : $1157-996 \mathrm{X}$

Référence électronique

Daniel Mouchard, «Réconcilier, réprimer : les "années de plomb" en Italie et les transitions

démocratiques dans le cône sud latino-américain », Cultures \& Conflits [En ligne], 40 | hiver 2000, mis en ligne le 28 septembre 2006, consulté le 30 mars 2021. URL : http://journals.openedition.org/ conflits/479; DOI : https://doi.org/10.4000/conflits.479

Ce document a été généré automatiquement le 30 mars 2021.

Creative Commons License 


\title{
Réconcilier, réprimer : les "années de plomb" en Italie et les transitions démocratiques dans le cône sud latino-américain
}

\author{
Daniel Mouchard
}

Réconcilier, réprimer: les "années de plomb» en Italie et les transitions démocratiques dans le cône sud latino-américain Sandrine LEFRANC et Daniel MOUCHARD Comment sortir d'une période de violence politique de grande ampleur et de la logique de «l'ennemi intérieur » qui, parfois, organise cette violence ? ${ }^{1} \mathrm{Si}$ l'on considère les modes volontaristes de «sortie» du conflit que peuvent adopter les gouvernements (et non la perspective d'une "usure temporelle »), deux solutions peuvent a priori s'offrir : soit le réprimer, voire l'éliminer, soit se réconcilier avec lui et le réintégrer à l'ordre social et politique. Si ces deux issues peuvent apparaitre disjointes, elles font pourtant toutes deux partie d'un continuum de stratégies politiques à des fins de "gestion» de l'ennemi intérieur; en ce sens, elles pourraient être complémentaires ${ }^{3}$. Ce sont ces enchevêtrements entre réconciliation et répression que nous tenterons d'éclairer. A cette fin, on s'appuiera sur les cas de deux processus politiques. Le premier de ces processus correspond à la répression militaire, puis aux logiques de "réconciliation $»^{4}$ politique dans le cône sud latino-américain dans le contexte de la transition des régimes militaires vers des gouvernements démocratiques (on peut dater approximativement cette période de 1973 à aujourd'hui). En Argentine, en Uruguay et au Chili, des coups d'Etat ont permis l'installation au pouvoir de régimes militaires au cours des années 1970, à la suite d'une exacerbation de la violence politique, en partie imputable à des mouvements armés d'extrême gauche (Tupamaros en Uruguay, Mouvement de gauche révolutionnaire [MIR] au Chili, Montoneros d'obédience péroniste et Armée révolutionnaire du peuple [ERP] en Argentine, parmi d'autres), ainsi que de tensions politiques venues fragiliser des systèmes politiques déjà instables $^{5}$. Ces régimes militaires, divers dans leurs formes, se sont caractérisés par un recours systématique à la répression, et notamment à la méthode de la disparition 
forcée ${ }^{6}$. Dans des circonstances variables, la délégitimation de ces régimes a rendu possible une transition négociée à la démocratie (en 1983-1984 en Argentine et en Uruguay, en 1989 au Chili); les termes de la transition étaient dans l'ensemble assez contraignants, les militaires conservant un pouvoir politique indéniable. Cette logique de la réconciliation consiste, d'abord, en des mesures de rémission. Le cas italien donne aussi à voir une considérable amplification de la violence politique, à partir du milieu des années 1970. C'est en effet dans le seconde moitié de cette décennie que l'activité des groupes armés d'extrême gauche (Brigades Rouges [BR], Prima Linea) se développe, les années 1978-1980 représentant le pic de cette violence, dont l'événement symbolique est l'enlèvement et l'assassinat d'Aldo Moro, retrouvé mort le 9 mai 1978. Mais la pénétration de la violence politique dans la société italienne ne se limite pas à l'activité des groupes militarisés : 1977 voit l'éclosion du mouvement des emarginati (exclus), où certains groupes d'extrême gauche (notamment Autonomie ouvrière, qui prend la relève des groupes en activité au début des années 1970, comme Potere operaio et Lotta continua) jouent un rôle important : cette extension de la contestation se caractérise par le développement de pratiques de violence «diffuse» (manifestations parfois armées, affrontements avec la police, «expropriations prolétaires »...), toutefois différente dans son intensité et sa nature de la violence exercée par les BR ou Prima Linea ${ }^{7}$. Face à ces différents types de violence politique, l'Etat italien répond par une répression unitaire, qui touche tous les groupes : le 7 avril 1979, de nombreux militants de l'Autonomie ouvrière (environ 70, dont les leaders Antonio Negri et Oreste Scalzone) sont arrêtés et inculpés pour faits de terrorisme, cette inculpation se fondant sur une identification entre Autonomie et BR. Les arrestations du 7 avril sont symboliques d'une phase de répression intense. A partir de 1978, différents dispositifs juridiques d'exception sont mis en place, qui se caractérisent essentiellement par une extension des pouvoirs de la police (interpellation, perquisition, garde à vue), et de la justice, ainsi qu'une réduction des droits des inculpés, observable notamment dans l'allongement considérable des durées de détention préventive ${ }^{8}$. Ainsi, les procès du « 7 avril 1979 » ne seront conclus qu'en 1984. L'effet de la répression sera à la mesure des dispositifs: durant la période de répression, 4087 militants seront condamnés et incarcérés. En 1997, on estime que 224 personnes sont encore incarcérées (dont 130 en régime de semi-liberté) et 190 en exil (la majeure partie en France). Il faut tout d'abord préciser le statut du rapprochement opéré entre le cas d'un régime démocratique (cas italien) et d'autoritarisme militaire (Amérique latine). Il ne s'agit pas, bien entendu, d'affirmer une équivalence qui écarterait le caractère discriminant du régime politique, mais de se concentrer sur un point commun bien délimité, et, peut-être, tirer sur ce point précis des enseignements d'ordre plus général et théorique. On ne parlera donc pas de comparaison mais de mise en parallèle, ou de mise en regard. L'analyse présentée ici n'est donc pas une analyse comparative stricto sensu, et laisse de côté un certain nombre de variables relatives aux différentes situations politiques, et de critères de comparaison. Cette importante restriction méthodologique permet néanmoins de se concentrer plus systématiquement sur une logique politique spécifique et sur ses implications théoriques. Nous partons en effet de l'hypothèse que les systèmes politiques que l'on examinera ici sont pareillement «travaillés » par la logique de l'ennemi intérieur, à la manière d'un cercle vicieux, c'est-à-dire une logique qui survit aux méthodes et discours politiques employés pour la dépasser (ou du moins qui se présentent comme tels). Cette logique de l'ennemi intérieur joue aussi bien, à des degrés certes fort 
différents, dans la démocratie en phase répressive des « années de plomb » italiennes et de leur suite, que dans les régimes militaires latino-américains en place depuis le milieu des années 1970 jusqu'aux décennies 1980-1990, et dans les démocraties nouvelles qui leur ont succédé dans le cône sud latino-américain. La question de la sortie de la logique de l'ennemi intérieur se pose dans les deux cas. Dans le cône sud latinoaméricain, la «réconciliation » passe prioritairement par l'amnistie, plus ou moins librement octroyée par les gouvernements démocratiques ou léguée par le régime autoritaire, des violations des droits de l'homme commises par les agents de l'Etat autoritaire dans le cadre de procédures de répression ; en Italie, au contraire, c'est la résolution des procédures judiciaires concernant la contestation et la violence dirigées contre l'Etat démocratique, la sortie des "années de plomb», qui est en jeu. Malgré l'évolution des pratiques et des discours concernant l'ennemi intérieur dans ces deux cas, nous postulons une continuité des représentations qui résiste plus ou moins aux changements politiques. Notons que le changement, dans les deux cas qui nous intéressent, n'est pas de la même nature: en Amérique latine, il s'agit bien d'une transition d'un régime autoritaire à un régime démocratique; en Italie, le régime ne change pas, mais le contexte politique se modifie profondément à partir de la fin des années 1970, notamment après l'assassinat d'Aldo Moro, dans le contexte de l'échec du compromis historique entre Démocratie Chrétienne et Parti Communiste Italien ${ }^{9}$. La dissemblance des processus engagés dans les deux cas par l'Etat doit d'abord être soulignée, pour pouvoir ensuite se concentrer sur la comparaison des traits communs. En Italie, l'Etat démocratique n'a pas construit de montage politico-juridique autonome de réconciliation, mettant seulement en place des procédures de rémission intimement liées aux processus de répression («repentir» et "dissociation»); le débat sur l'amnistie, plusieurs fois lancé, n'a jusqu'ici pas abouti. Que l'amnistie soit ou non souhaitable sur un plan normatif (ce qui n'est pas le propos de ce texte), ces impasses juridiques montrent que les représentations qui sous-tendent ce processus, dépendantes de la logique de l'ennemi intérieur, n'ont pas disparu. Dans les trois pays du cône sud latino-américain, la "réconciliation" démocratique a succédé à la répression totale, brutale (à la fois légale et, surtout, illégale) des régimes militaires. Des lois d'amnistie ont été adoptées ou entérinées (après, en Argentine, un processus très sélectif de poursuites judiciaires, et alors que certaines poursuites résiduelles ont encore lieu, de même qu'au Chili). L'ennemi du régime militaire, le «subversif » ou «terroriste $»^{10}$, est devenu la victime. L'Etat criminel ${ }^{11}$ a échappé, semble-t-il, à sa désignation comme nouvel ennemi intérieur; demeure un corps militaire en partie professionnalisé. Ces évolutions ont lieu sur fond d'une rhétorique de la réconciliation déployée par l'Etat démocratique, qui, si elle ne provoque pas forcément l'adhésion des deux parties anciennement (et encore aujourd'hui dans les faits) adverses (terroristes contre militaires, puis victimes contre militaires), est en grande partie, semble-t-il, ratifiée par la majorité de la population ${ }^{12}$. Il s'agirait là, a priori, de deux logiques antithétiques, de deux modalités contradictoires de résolution de la question de l'ennemi intérieur par l'État ${ }^{13}$ : en Italie, une logique d'affrontement binaire, en Amérique latine, un Etat prétendant au rôle de tiers réconciliateur. Or l'absolutisation de cette différence, qui permet de valoriser le modèle de la réconciliation comme modalité idéale, sinon exclusive, de gestion/dépassement de la logique de l'ennemi intérieur ${ }^{14}$, nous semble conduire à une compréhension incomplète des phénomènes en jeu. D'une part, considérer qu'il s'agit de deux modèles radicalement différents permet de faire l'économie d'une étude de la "réconciliation », en posant que celle-ci, par une 
sorte de miracle performatif (qui est précisément celui attendu par les tenants du discours de la réconciliation, faute de pouvoir agir directement sur les représentations collectives, et sur les haines et ressentiments individuels), permet une réconciliation effective. Or, les politiques "réconciliatrices" dans le cône sud latino-américain semblent ne pas avoir permis une réconciliation. On n'entend pas signifier ici l'inéluctabilité d'un échec du modèle de la réconciliation ${ }^{15}$, mais insister sur l'intérêt d'une analyse des "ratés" locaux de la réconciliation. D'autre part, poser une opposition entre réconciliation et répression ne permet pas de prendre en compte certains processus discursifs et pratiques communs aux deux modalités, qui sont révélatrices des difficultés extrêmes à sortir de la logique de l'ennemi intérieur. En effet, on pose ici comme hypothèse que la réconciliation découle d'une volonté de restauration de l'ordre politique (sinon de l'ordre en général), de restauration d'une unité nationale, de «normalisation» des violences (pacification), afin de faire réapparaître les possibilités d'un jeu politique et institutionnel normalisé (ce qui transparaît dans l'idée de compromis avancée par les «transitologues $\left.{ }^{16}\right)$. Dans ce contexte, les politiques volontaristes de réconciliation supposent donc une fixation des identités, une reconnaissance des culpabilités, et l'élaboration d'un ordre du discours unifié $^{17}$. C'est sur ces processus communs à la réconciliation et à la répression que nous allons tenter d'insister. Logique de l'ennemi intérieur et recherche d'unité politique La proximité relative des modèles de la réconciliation et de la répression prend racine dans la parenté des périodes de crise, de violence politique généralisée, qu'ont connues l'Italie et les pays du cône sud latino-américain, toutes proportions gardées. Ces deux modèles reflètent l'étroite articulation de la construction d'un ennemi intérieur (il s'agit bien de construction plus encore que d'une simple nomination ou désignation) et de la recherche plus ou moins explicite d'une unité politique, incontestable, intouchable. Amérique latine et Italie: des modalités communes de désignation de l'ennemi intérieur Dans les deux cas qui nous occupent, la gauche " radicale » ou extraparlementaire est représentée comme l'agent de subversion, de l'ordre démocratique et du «compromis historique » en Italie, de l'ordre "chrétien» et libéral (au sens économique du terme) revendiqué par les militaires en Amérique latine. La situation est apparemment inversée: ceux qui sont jugés en Italie correspondent, par leur idéologie et leurs pratiques politiques, à ceux au nom desquels on dit choisir de ne pas juger dans le cadre de la "réconciliation» démocratique en Amérique latine (les victimes anciennement affiliées aux terroristes). Cependant, cette inversion ne doit pas occulter la parenté qui lie les systèmes d'opposition entre Etat et mouvements radicaux ${ }^{18}$. Du «terroriste» au "subversif $~^{19}$ : la logique expansionniste de la désignation de l'ennemi intérieur est comparable en Italie et en Amérique latine. Même si, dans le cas italien, on ne peut dissocier absolument les pratiques de violence politique organisée, les pratiques contre-culturelles associées à des actes violents ponctuels de "banditisme social», et les discours théoriques qui les entourent et les légitiment ${ }^{20}$ (ceci étant dû à la structure diffuse et complexe de l'« aire » de l'autonomie en $1977^{21}$ ), il ne subsiste pas moins des différences cruciales, notamment au niveau de structures organisationnelles et de l'usage de la violence qui en découle (structure léniniste/modèle militarisé des BR, modèle décentré/violence «diffuse» pour l'Autonomie ouvrière [AutOp]). Les relations entre les deux sont complexes, entre passages (AutOp vers BR) et inimitié (les BR parlent des «eaux troubles de l'Autonomie ", et de violentes critiques à l'égard des BR émanent de l'Autonomie à partir de 1978). En tout état de cause, on note dans les deux cas une tendance du régime 
à passer outre les seuils existant entre les mouvements armés (Brigades rouges, Prima Linea... en Italie, et MIR, Montoneros, Armée révolutionnaire du peuple [ERP], Tupamaros dans le cône Sud) et l'acteur ayant une proximité idéologique forte. C'est l'opération réalisée par l'« hypothèse Calogero » (ou "théorème Calogero ", du nom du procureur général de Padoue) postulant une équivalence entre activité terroriste et production intellectuelle/politique (théorie de la «vitrine légale») : en vertu de ce théorème, Antonio Negri, intellectuel impliqué dans l'Autonomie ouvrière, se voit fortement suspecté (ce soupçon étant transformé en quasi-certitude par la focalisation judiciaire et médiatique) d'avoir assumé la direction intellectuelle et politique des BR, cette assertion étant appuyée par les confessions du «repenti » Carlo Fioroni en décembre 1979, qui fait état de rencontres entre Negri et Renato Curcio (fondateur des $\mathrm{BR}$ ), et de la constitution d'un «bras armé » de Potere operaio (organisation préalable à l'Autonomie, dont Negri fut un des principaux dirigeants), qui aurait fait le lien avec les BR. De cette "Organisation", on ne trouve pourtant pas de trace irréfutable. Le témoignage de Fioroni sera d'ailleurs remis en cause, et Negri innocenté assez rapidement (en avril 1980, en même temps que Franco Piperno), pour les charges relatives à l'assassinat de Moro. Mais d'autres charges pèsent encore à ce moment sur lui, et de nouvelles s'ajouteront ultérieurement, reconduisant la logique d'assimilation autonomie-terrorisme, qui est bien exprimée par cette déclaration du sous-secrétaire d'Etat à l'Intérieur Mazzola (Démocratie chrétienne [DC]), en 1980: «Les collectifs autonomes comptent 100000 sympathisants, on peut donc considérer qu'il y autant de terroristes potentiels ». En Amérique latine, si l'intensité et la nature de la répression mise en œuvre par les régimes autoritaires est évidemment sans commune mesure avec la démocratie italienne, on peut néanmoins comparer la logique de désignation de l'ennemi intérieur, en ce qu'elle relève d'un processus comparable de dilatation et d'extension de l'ennemi : la répression militaire vise les terroristes d'abord, leurs «idéologues » ensuite, sinon tous les intellectuels voire leurs proches. On peut citer quelques exemples, qui, s'ils se situent dans le cadre d'une répression beaucoup plus radicale qu'en Italie, participent, toutes proportions gardées, d'une même logique de dilatation de la figure de l'ennemi intérieur. Au Chili, le général Pinochet déclare le 11 septembre 1977: «Le marxisme est une doctrine intrinsèquement perverse ». De manière plus explicite encore, on peut citer le général chilien Horacio Toro $^{22}$, précisant que les militaires "entraient en guerre contre le marxisme, et la chose la plus importante était d'anéantir, je dis bien d'anéantir, le marxisme, et non restaurer la démocratie. Et l'anéantissement du marxisme ne pouvait avoir lieu dans un climat démocratique ; l'anéantissement du marxisme et de tous ceux qui se considéraient promarxistes, neutres, tièdes, indéterminés, a été mené avec des méthodes brutales de répression qui n'avaient pas de limites ». Pour l'Uruguay, même si l'anéantissement physique n'était pas la priorité, un habitant sur six pourrait avoir été emprisonné entre 1972 et 1983. Enfin, l'Argentine pousse cette logique à son paroxysme. Citons le général Ibérico Saint-Jean, gouverneur de Buenos Aires en 1978: « Nous tuerons d'abord tous les subversifs; puis nous tuerons ceux qui ont collaboré avec eux; ensuite, nous tuerons ceux qui sont restés indifférents, et, enfin, nous tuerons les timides ». Une logique bipolaire au service de l'unification La logique de l'ennemi intérieur peut se comprendre comme une logique bipolaire exacerbée ${ }^{23}$. Cette logique entraîne des effets de radicalisation de l'affrontement, notamment de la part de l'Etat. En Amérique latine, cette logique est légitimée par la Doctrine de sécurité nationale, qui oppose le rétablissement d'un ordre "chrétien " et libéral à une "conspiration communiste 
internationale». Notons toutefois que cette Doctrine peut se décliner selon deux versions au moins : une version " forte » ne tolérant pas de position de neutralité entre le «nous » de l'ordre et le « eux » subversif, et une version «pragmatique » tolérant, sinon recherchant un point neutre, malléable, et idéologiquement indifférent, pour obtenir une stabilisation de l'ordre autoritaire ${ }^{24}$. En Italie, le processus d'affrontement BR-Etat se situe aussi dans ce schème bipolaire et progressivement radicalisé, qui serait peu à peu entraîné dans cette relation spéculaire entre terrorisme militarisé et répression étatique. L'enlèvement et l'assassinat d'Aldo Moro (président de la DC et acteur essentiel du "compromis historique»), en 1978, sont le point nodal de cette bipolarisation-focalisation des deux parties ${ }^{25}$, en fonction de la stratégie des BR (visant à provoquer un soulèvement populaire), et du raidissement de l'Etat et des partis durant cette affaire ${ }^{26}$. Des intellectuels proches de l'autonomie (dont Paolo Virno ${ }^{27}$ ) expriment, dans leur interprétation de cette période, la perception de cette réduction, de cette simplification de la logique conflictuelle, qui tend du même coup à occulter la spécificité revendicative et organisationnelle de la mouvance autonome (au profit, si l'on peut dire, d'une vision exclusivement « léniniste » des schèmes organisationnels). Cette bipolarisation/radicalisation a deux effets : d'une part, comme on l'a vu, elle offre un terrain favorable pour une constitution "unifiante» de la figure de l'ennemi intérieur (c'est précisément sur ce point que porte la critique de Virno). D'autre part, elle rend possible une expansion de la violence d'Etat à travers un dispositif juridique ad hoc. Résumons rapidement ce dispositif ${ }^{28}$ : loi Reale de mai 1975 augmentant les pouvoirs de la police (permettant notamment les arrestations sur " intime conviction » sans mandat du juge d'instruction), décret-loi de mars 1978 (qui étend encore les pouvoirs de perquisition et d'arrestation), et les deux lois Cossiga de décembre 1979 et février 1980, qui outre les dispositions sur le repentir (sur lesquelles nous reviendrons), introduisent de nouveaux chefs d'inculpation («terrorisme », et « insurrection armée contre les pouvoirs de l'Etat »). Dans ce dispositif, plusieurs points témoignent à la fois de la «délégalisation " de l'État ${ }^{29}$ et de ce qui nous intéresse particulièrement ici, à savoir la logique expansionniste de désignation de l'ennemi intérieur : en particulier, le caractère rétroactif de plusieurs dispositions juridiques (qui permettent aussi de nouvelles qualifications des personnes), la définition hasardeuse des délits et l'invention de nouveaux (la notion de "crime odieux" dans l'avis d'extradition de Franco Piperno, celle de "concours moral ", lors du procès de Turin, en 1981). La mention d'«actes préparatoires " (atti preparatori) dans la loi Reale ${ }^{30}$ ou, dans un décret de 1979, une disposition selon laquelle toute personne qui possède, imprime et diffuse des documents à caractère terroriste peut être inculpée, se situent encore dans cette logique ${ }^{31}$. Ces dispositifs juridiques contribuent à la bipolarisation, la cristallisation des figures et des identités en deux camps opposés. La constitution de l'ennemi intérieur, c'est la désignation par l'Etat d'un "autre ", d'un corps étranger, porteur intérieur d'une extériorité radicale qui passe pour menacer l'unité et la sûreté de la société. De fait, cette qualification, cette désignation permettent en retour une affirmation à vocation performative de l'unité du «corps» social et politique ${ }^{32}$. Du point de vue de cette recherche d'unité, la position d'un ennemi intérieur permet le rapprochement de l'Etat et de certaines parties de la société et du champ politique : de ce point de vue, l'attitude du Parti communiste italien, «parti d'opposition mais parti d'ordre ", qui soutient activement les mesures répressives ${ }^{33}$, même après l'échec du " compromis historique », et qui garde à l'esprit un « horizon d'unité nationale » (selon les termes de Berlinguer) est significative. Cette logique permet aussi d'occulter les 
faiblesses de l'Etat et la conflictualité sociale. En Italie, selon l'analyse d'Isabelle Sommier ${ }^{34}$, la faiblesse historique de l'Etat est un facteur explicatif déterminant de la montée de la violence politique. Cette faiblesse est encore renforcée par l'instabilité politique qui fait suite à l'assassinat de Moro: il est significatif que le personnage représentatif de la force de l'Etat soit à ce moment-là un militaire, le général Dalla Chiesa $^{35}$. En Amérique latine, la Doctrine de la sécurité nationale et, plus généralement, les discours politiques produits par les régimes militaires, montrent bien cette imputation à l'autre de la production de la violence et de la division, et du même coup, l'occultation de la conflictualité sociale par la construction de l'Etat (et des forces armées) comme une figure tutélaire et unifiée ${ }^{36}$ : «Le pays et la nation entiers constituent un seul bloc, une seule armée. La guerre impose aujourd'hui une unité absolue, la cohésion parfaite du pays ${ }^{37}$. C'est précisément cette logique d'unification qui explique que, au-delà des différences fondamentales entre contexte italien et contexte latino-américain, répression et réconciliation mettent en œuvre certains processus discursifs et pratiques communs. C'est ce que nous allons essayer de démontrer, en prolongeant la comparaison vers les suites, sinon la sortie, de la répression, dans les deux contextes qui nous occupent: d'une part, le modèle de la « réconciliation » démocratique en Amérique latine (à partir de 1983); d'autre part, le débat autour de la "fin des années de plomb» en Italie, qui se développe dans les années 1980. Réconciliation et répression : une même logique d'unification politique La "réconciliation" latino-américaine: du pluralisme proclamé à la réunification En Amérique latine, dans le cadre du processus de transition démocratique, la volonté déclarée de restauration du pluralisme (selon un modèle de démocratie libérale), tant au sein du système politique que de la sociétée ${ }^{38}$, se présente comme une tentative de dépassement de la logique de l'ennemi intérieur. De ce point de vue, la tentative de transposition dans le système politique d'une relation de pardon (telle qu'elle est notamment définie dans la théologie et la philosophie morale) est hautement significative. Or, ce modèle de la réconciliation semble échouer au sens où, dans cet horizon de règlement du conflit qu'il pose, la conflictualité n'est pas reconnue, pas assumée: la réconciliation s'enlise dans une logique d'évitement du conflit, et reconduit donc finalement les postulats d'unité a priori qui caractérisaient déjà le régime militaire. Sans entrer dans les détails, cette logique d'évitement est reconduite à un triple niveau. Un niveau politique, d'abord : la recherche d'un compromis entre les diverses parties (entre les responsables du régime militaire et les responsables du nouveau gouvernement démocratique ${ }^{39}$, anciens opposants, voire entre ces derniers et les victimes de la répression militaire), qui alimente un discours prônant la réconciliation entre victimes et bourreaux, est privilégiée. Un niveau juridique et institutionnel, ensuite : il existe, entre les régimes militaires et démocratiques, une forte continuité juridique des régimes (amnistie votée durant le régime militaire et entérinée par le régime démocratique ou amnistie adoptée par le régime démocratique; principe de non-rétroactivité interdisant de créer de nouveaux chefs d'inculpation pour certaines pratiques répressives, dont les « disparitions »; continuité légale, institutionnelle, et administrative). Un niveau « idéologique », enfin : le discours sur la réconciliation est toujours fondé sur une référence tautologique à l'unité nationale, à la fois postulat et objectif de ce discours. On retrouve ici la définition de la réconciliation que l'on a précédemment esquissée : la réconciliation suppose une mise en ordre et une normalisation unifiante. En ce sens, l'objectif affiché de pluralisation et la logique d'unification intrinsèque font de la réconciliation un concept contradictoire, 
tout au moins dans son application politique (un exemple de mise au jour de cette contradiction est le refus de la part des victimes d'accepter une réconciliation imposée et homogénéisante ${ }^{40}$. La répression italienne : un processus de réconciliation bloqué ? En Italie, le blocage du processus de sortie de la répression se manifeste clairement par l'enlisement (les délais de détention préventive s'étendent ${ }^{41}$ ) et la prolifération, incroyablement complexe, des procédures judiciaires. Ainsi, pour ne prendre que le cas d'Antonio Negri, les chefs d'inculpation à son encontre se multiplient et se "dilatent »: il sera successivement inculpé d' " association subversive", de " constitution de bande armée ", d'« insurrection armée contre les pouvoirs de l'Etat » (chef d'inculpation créé par la loi Cossiga de 1979), de "responsabilité morale " dans les affrontements entre militants d'extrême gauche et forces de police qui ont marqué les années 1970, ainsi que, comme on l'a vu, d'une responsabilité dans l'assassinat d'Aldo Moro. Certains de ces chefs d'inculpation ont été progressivement abandonnés, d'autres persistent jusqu'à aujourd'hui, même s'ils sont allégés et s'il a rapidement bénéficié de la liberté conditionnelle (notons que ce n'est pas le cas de nombreux militants d'extrême gauche emprisonnés). Dans le même ordre d'idées, le phénomène d'exil (ou d'« expatriation ») politique de membres de l'extrême gauche italienne, durant les années 1980, notamment en France, montre bien ce blocage de la réconciliation, ou, en tout cas, d'une solution gouvernementale "volontariste». Ces deux phénomènes peuvent s'analyser comme des phénomènes d'exclusion tant intérieure pour les emprisonnés (c'est d'ailleurs le terme qu'ils utilisent ${ }^{42}$ ), qu'extérieure, pour les exilés. D'autres éléments corroborent cette constatation du blocage du processus (si on exclut les grâces présidentielles accordées au cas par cas). L'échec du débat, relancé en 1987 par Curcio et Moretti (leaders historiques des BR), autour d'une possible amnistie manifeste cette continuité et ce blocage sur la logique répressive. Un verrouillage s'opère quand Bettino Craxi fait voter une loi exigeant une majorité des deux tiers à la Chambre et au Sénat pour une loi d'amnistie. Notons que ce refus de l'amnistie comme règlement politique semble être une caractéristique plus générale du processus politique italien ${ }^{43}$. En 1997, ce débat connaît une résurgence suite à une proposition de Rifondazione communista, mais ne connaît pas de suite. Ces différents débats manifestent donc des tendances, des tensions vers la solution "réconciliatrice", mais qui restent bloquées. On peut avancer des éléments d'explication de ce blocage dans le cas italien: c'est, comme on l'a déjà souligné, la faiblesse historique de l'Etat - sa difficile unification - et $\mathrm{du}$ système politique italiens qui peuvent en dernière instance expliquer cet état de fait. Elle rend problématique le postulat d'unité que suppose la réconciliation, et éventuellement, l'amnistie, si on considère que l'amnistie suppose une unité forte pour éviter une déstabilisation du système politique (unité que la répression, elle, impose unilatéralement). Plus précisément, de ce point de vue, la persistance de la logique répressive en Italie et l'échec du débat sur l'amnistie durant les années 1980 peuvent être reliés à un état de crise du système politique durant cette période (sans que, comme on le verra, cela constitue le seul facteur explicatif). Si l'amnistie peut être une arme stratégique de l'Etat dans une situation de confrontation directe avec les groupes armés, et donc utilisée avec parcimonie, le blocage de cette voie, alors même que des pressions se manifestent, une fois que le temps de la confrontation directe est révolu, suscite des interrogations. Ce blocage témoigne alors de la rémanence de la logique de l'ennemi intérieur. Cette rémanence s'enracine dans une différence quantitative évidente entre les deux contextes étudiés ici, qui renforce le facteur étatique: en Amérique latine, les agents de la violence d'Etat sont un groupe à la fois 
quantitativement important et intégré à l'appareil étatique, encore institutionnalisé après la transition (en tant que forces armées). De ce fait, l'hypothèse d'un jugement de masse se heurte à la fois à la faiblesse et à l'illégitimité de l'appareil judiciaire, au coût d'une telle justice collective, et à la menace persistante que représentent les forces armées pour la démocratie nouvellement installée (le « chantage au putsch»). De plus, remonter la chaîne des responsabilités pourrait supposer la mise en cause de nombreux groupes sociaux («complices» du régime militaire), ce qui augmenterait encore les risques de déstabilisation globale. Au contraire, en Italie, le groupe considéré est beaucoup plus faible numériquement, et n'est pas intégré à l'appareil d'Etat: la possibilité d'un jugement collectif est donc beaucoup plus aisément réalisable, y compris dans sa forme extensive : on estime aujourd'hui le nombre d'emprisonnés à environ 180 et le nombre d'exilés, principalement en France, à environ 150. En Italie, donc, le jugement ne remet pas en cause les fondements de l'ordre démocratique (l'Etat peut donc déployer une logique répressive unificatrice sans prendre le risque d'être déstabilisé par le nombre), alors que, dans le cas latino-américain, la menace d'un putsch militaire est présente et parfois bien réelle ${ }^{44}$. Réconciliation et répression: l'imposition unilatérale d'une parole d'Etat? Répression et réconciliation : l'ordre du discours étatique Découlant directement du processus de répression, le phénomène des «repentis » en Italie (encadré juridiquement par les lois Cossiga) témoigne d'une volonté d'annexion par l'Etat du discours autre à l'ordre du discours étatique. Les processus d'extorsion de l'aveu sont une modalité spécifique d'exercice du pouvoir : ce qui est exigé dans le repentir, c'est le renoncement à la violence, et l'acceptation de la légitimité du régime. Comme le dit Michel Foucault, dans La Volonté de savoir : «l'aveu de la vérité s'est inscrit au cœur de procédures d'individualisation par le pouvoir »; le "vieux modèle juridico-religieux de l'aveu» participe de l'assujettissement des hommes, de «leur constitution comme "sujets", aux deux sens du mot $»^{45}$. Le repentir peut donc apparaître comme un complément à la répression frontale, cela d'autant plus que, dans son acception littérale, il fait signe vers la réconciliation. D'où l'idée que les phénomènes de répression directe et le repentir réconciliateur sont liés organiquement : le repentir sert la répression par les informations qu'il fournit, mais, plus encore, repentir et répression sont deux modalités complémentaires de l'exercice du pouvoir d'Etat et de la gestion de l'ennemi intérieur. On peut là encore se référer à Foucault et à la distinction qu'il opère entre modèle belliciste et modèle pastoral du pouvoir ${ }^{46}$. Le phénomène du "repentir» connaît une forme de contrepartie dans le cône sud latino-américain: il s'agit du repentir a priori librement exprimé par des «francs-tireurs » du remords. Le remords individuel n'a en effet pas sa place dans la réconciliation décrétée par les gouvernements démocratiques latino-américains, alors qu'il tient une place importante, sous la forme d'une « confession » exhaustive qui peut ne pas être associée au remords, en Afrique du Sud. La révélation complète des crimes politiques commis par l'offenseur conditionne l'obtention de l'amnistie. En Amérique latine, au contraire, si la demande de pardon des responsables de violations des droits de l'homme semble être appelée par le discours sur la réconciliation nationale, les rares expressions individuelles de repentir (la confession du capitaine Adolfo Scilingo ${ }^{47}$, en Argentine) viennent troubler l'ordre institué par la politique de " justice de transition » des gouvernements démocratiques et représentent une forme très relative de rébellion contre l'institution militaire (Scilingo avait été « lâché » par les forces armées). La seule forme de repentir apparemment compatible avec la logique de réconciliation mise en œuvre dans le cône sud latino-américain semble être la "repentance institutionnelle » 
(exprimée par l'Eglise et par les forces armées argentines, par exemple), qui traduit un repentir très relatif ${ }^{48}$ et une réintégration dans l'ordre institutionnel et normatif d'une démocratie en voie de consolidation. La logique de réconciliation décrétée par le gouvernement n'appelle pas un repentir en acte ou une relation de pardon, alors qu'elle recourt au registre religieux et moral du pardon: elle semble plutôt le bannir, parce qu'il pourrait troubler l'ordre institué. Ainsi, qu'il s'agisse de la répression ou de la réconciliation, le repentir n'a sa place que lorsqu'il participe de l'imposition unilatérale d'une parole d'Etat, d'une normalisation. Il est converti en procédure judiciaire parce qu'il permet de renforcer une "nouvelle » assignation des rôles par l'Etat (le terroriste est exempté de peine, « réintégré » dans la société), qui participe en fait d'une stigmatisation renouvelée de l'ennemi intérieur (celui qui ne se repent pas); son expression individuelle est paradoxalement malvenue dans les processus de réconciliation latino-américains, dans la mesure où il vient troubler la logique politicojuridique, "rouvrir les plaies» qui ont été officiellement fermées. Le discours judiciaire, et celui des autorités exécutives dans les « attendus » des textes de loi et des décrets et le discours des magistrats, participe d'une même imposition de la parole univoque de l'Etat. En Italie, la volonté d'attribution d'une responsabilité aux dirigeants intellectuels de l'Autonomie ouvrière entraîne la construction, par les juges et les procureurs, d'un discours qui traduit une confusion du "dire» (productions intellectuelles) et $d u$ «faire» (actes violents dont il est possible de faire la preuve matérielle), au sens non où il pénalise l'incitation à la violence (incrimination classique), mais où il donne à l'incitation le statut légal de participation aux faits. Il est frappant, dans les extraits de l'interrogatoire de Negri en 1979 dont on peut disposer ${ }^{49}$, de constater que l'administration de la preuve par le juge et le procureur reste hésitante, et qu'elle se fonde surtout sur des procédures répétitives et performatives, parfois à visée déstabilisatrice ${ }^{50}$, face aux explications théoriques de Negri ${ }^{51}$. Les propos de l'autorité judiciaire à cette période corroborent cette incertitude du discours juridique, comme le montrent les propos du procureur général Guasco (de Rome), qui déclare qu' "étant donné que nous avons affaire à des organisations clandestines, il est très difficile de trouver des preuves " (pourtant des "preuves ", mais sous forme de documents théoriques, sont citées abondamment lors de l'interrogatoire de Negri), et que « le magistrat doit exprimer sa propre conviction à partir de l'interprétation des documents et des témoignages $\aleph^{52}$. En Amérique latine, la rareté des poursuites judiciaires n'empêche pas une attribution des responsabilités, essentiellement par les autorités exécutives. Une logique minimaliste prédomine (en matière d'inculpations et a fortiori de poursuites: en Argentine, seuls les commandants en chef et quelques autres officiers haut-gradés ont fait l'objet de poursuites en 1985; au Chili, les poursuites ne sont possibles que pour les délits relativement rares qui ne sont pas couverts par l'amnistie de 1978) ${ }^{53}$. Cependant, au contraire de l'Italie, un principe d'équivalence est posé entre les anciens terroristes et les responsables militaires de violations des droits de l'homme : la «théorie des deux démons » du gouvernement Alfonsín en Argentine justifiait, dès 1984, des décrets présidentiels ordonnant des poursuites symétriques; on trouve en Uruguay et au Chili des perceptions similaires. Le gouvernement démocratique infirme les interprétations historiques des militaires, donne aux proches des anciens « subversifs » sinon aux «subversifs » eux-mêmes un statut de victimes, sans pour autant officialiser les interprétations historiques de ces derniers, puisqu'il choisit de mettre au même niveau de responsabilité les deux formes de violence politique. Le discours judiciaire permet ainsi de réduire la complexité de la 
réalité des délits commis pour consolider le discours de l'Etat et reproduire une répartition bipolaire des sujets soumis à la "justice de transition ». Les identités assignées à ces sujets ne sont qu'en partie reformulées de manière à traduire le changement politique et le recouvrement d'une « vérité historique » officielle (par les rapports des « commissions de vérité et de réconciliation ») : l'ancien «subversif », ou ses proches dans le cas d'une disparition ou d'un assassinat, deviennent les victimes, mais les forces armées n'en deviennent pas pour autant les coupables identifiés ${ }^{54}$. Pour autant, outre que cette assignation des rôles reproduit à sa manière une logique bipolaire, les étiquettes attribuées sont souvent floues (les victimes sont aussi en partie des coupables - responsables de l'émergence de la violence politique - les coupables ne sont pas tout à fait des coupables...). Entre ces deux parties, l'Etat (les gouvernements démocratiques) joue un rôle qui peut apparaître "schizophrène » : du côté des victimes au nom de la vérité historique et, parfois, d'une ancienne proximité idéologique et politique, du côté des "coupables" au nom de la consolidation démocratique et de l'appartenance des forces armées à l'Etat, il ne parvient jamais tout à fait à remplir le rôle de tiers arbitre qui reviendrait à l'Etat justicier - au sens non d'une abstention justifiée par l'impartialité, mais du rôle de garant de la justice. Les victimes et les "coupables» s'enlisent ainsi dans une relation de ressentiment toujours répété, marquée par le refus du pardon par la plupart des victimes, parce que celui-ci n'est pas demandé ou dans son principe même, parce qu'il implique une "perversion du langage » de la justice ${ }^{55}$, et par le refus de la demande de pardon et de la reconnaissance de l'ancien ennemi, toujours suspect, par les militaires. L'Etat instaure donc un ordre du discours, qui est le soubassement des procédures de désignation, de répression, de l'ennemi intérieur. Or, la structure de cet ordre du discours influe sur la possible " sortie » de la logique de l'ennemi intérieur, telle qu'elle est mise en œuvre par les « interlocuteurs » de l'Etat. Les stratégies ambivalentes des « exclus » : entre extériorité et « réconciliation » Les stratégies étatiques de désignation, de répression de l'ennemi intérieur ont pour conséquences des processus de stigmatisation et d'exclusion de ce groupe ennemi ${ }^{56}$. En Italie, la stigmatisation est particulièrement forte vis-à-vis de certains individus, dont Toni Negri, assimilé à une figure démoniaque ${ }^{57}$. Plus généralement, c'est le stigmate de la violence, stigmate qui, comme on l'a vu, s'applique à différentes catégories de personnes, qui remplit la fonction d'étiquetage. A partir de cet étiquetage, l'exclusion de la communauté démocratique se manifeste concrètement par deux phénomènes : l'emprisonnement de longue durée (les « exclus de l'intérieur ») et l'exil politique pour un certain nombre d'intellectuels et de militants (dont Toni Negri et Oreste Scalzone). L'extériorité de l'ennemi intérieur apparait donc de manière physique. Notons que cette extériorité radicale apparaît évidemment comme un facteur de blocage pour un processus de réconciliation, qui suppose au moins une co-présence des acteurs, sur un terrain discursif commun. D'une manière similaire à certains égards, les Mères de la Place de mai (Madres de Plaza de Mayo), regroupement des femmes, des sœurs, et surtout des mères des disparu(e)s, né vers 1976 en Argentine, ont été étiquetées comme "folles", par le régime militaire et ont fait l'objet d'une répression (interdiction, harcèlements, et «disparitions» suite notamment à l'infiltration du commandant Astiz). Une fois la démocratie restaurée, elles sont devenues les victimes "légitimes» de la répression ${ }^{58}$. Or, leur refus de toute " réconciliation », traduit par la radicalité de leurs slogans (Aparición con vida, c'est-àdire l'exigence, volontairement contre toute vraisemblance, d'un « retour en vie » des disparus, slogan qui se traduit par le refus des déclarations légales de décès, des 
exhumations et des indemnisations par l'Etat), et par leur réticence à l'égard des politiques qui étaient a priori les plus conformes à leurs revendications (tels la CONADEP, la commission de vérité argentine, et le procès des juntes), les ont fait basculer progressivement dans une nouvelle position d'extériorité, sinon d'exclusion. Ce retranchement volontaire a été à certains égards entériné par les gouvernements démocratiques (pour lesquels elles représentaient une forme d'opposition, d'autant plus qu'une partie des Mères a revendiqué un rôle explicitement politique): elles n'étaient plus «folles » peut-être, mais elles restaient « déraisonnables », représentant une menace pour l'ordre politique (par leur refus du compromis et de l'ordre, par le brouillage des sphères publique et privée dont elles étaient responsables). Là encore, même si les situations diffèrent, et que les groupes considérés n'ont pas le même statut, on peut comparer les logiques d'étiquetage, de stigmatisation, et éventuellement d'exclusion qui sont à l'œuvre. Cette position d'extériorité n'est qu'une des composantes de la stratégie des acteurs face au discours d'Etat. Si la position des Mères de la Place de Mai n'était déjà pas dénuée d'ambivalence, une grande partie des victimes et organisations de défense des droits de l'homme latino-américaines ont pris le parti d'une position plus nuancée à l'égard des politiques gouvernementales œuvrant de ce fait à une "réconciliation ${ }^{59}$. Dans le cas de l'Italie, l'extériorité n'est pas une stratégie délibérée au départ. Les stratégies qu'il faut considérer ici, ce sont celles qui sont développées à partir de cette position d'extériorité. On peut considérer que les phénomènes de repentir et de dissociation, conséquence de la stratégie étatique de constitution de l'ennemi intérieur et des stratégies qui y réagissent, constituent des blocages à une possible réconciliation (la "solution de compromis", opposée à la solution répressive). Repentir et dissociation sont deux procédures différentes: le repentir, procédure individuelle, consiste en un échange entre informations et remise de peine. La « dissociation », procédure initiée par l'« appel des 51 » en 1982, et codifiée juridiquement en 1987, n'implique pas de dénonciation, mais une reconnaissance de l'erreur constituée par la lutte armée et la renonciation à l'utilisation de la violence : pour bénéficier de la remise de peine, le "dissocié » doit affirmer la réalité de cet engagement par son comportement. Sans chercher à approfondir les différentes motivations des acteurs de ce processus (la procédure de "dissociation " provoquera des conflits au sein de la communauté carcérale), on peut noter que repentir et dissociation participent du même phénomène d'annexion de la parole des "exclus » à l'ordre du discours étatique. On peut d'ailleurs constater que certains intellectuels ayant appartenu à la "mouvance" autonome qui critiquent les phénomènes de repentir sous ce motif $f^{60}$, reconduisent néanmoins l'argument de la dissociation. La manifestation de ce phénomène d'annexion au discours étatique est l'incapacité de ces procédures à déboucher sur une solution de compromis non judiciaire ${ }^{61}$. A cet égard, le cas de Toni Negri est particulièrement significatif. Il est un des initiateurs de la stratégie de dissociation. Néanmoins, il choisit l'exil et se réfugie en France. Les textes écrits en exil peuvent être lus comme un pas supplémentaire à partir de la logique discursive de dissociation, en appelant à une refondation démocratique radicale, devant se traduire notamment par la réconciliation et l'amnistie. L'appel à la refondation vise en même temps à « clore » le phénomène de la violence politique. ${ }^{62}$. Le retour de Negri en Italie en 1997 pour se constituer prisonnier prolonge cette logique de «refondation »: utiliser la position d'extériorité (c'est-à-dire, ici, le transfert de l'exil à la prison) pour promouvoir une stratégie de réconciliation. Mais, de fait, cette stratégie est jusqu'ici restée inefficace : cet échec peut en partie être expliqué par la 
confusion discursive et politique créée par les différentes procédures de rémission/ répression mises en œuvre conjointement durant la décennie $1980^{63}$. Au terme de cette courte étude, qui n'épuise évidemment pas les difficultés de la mise en regard et qui ne prétend pas donner les clés ultimes de compréhension des situations étudiées, on peut néanmoins s'autoriser quelques conclusions fort générales. Ce que notre étude a peutêtre contribué à montrer, c'est la difficulté du passage d'un ordre politique à un autre. Pourquoi ? Parce que tout ordre politique est sous-tendu, structuré par un système d'identités progressivement cristallisées et/ou assignées, un «schéma actantiel » qui fige les acteurs dans des rôles, et fige les relations entre eux. De cet ordre et du schéma qui le structure, il est difficile de s'extraire, notamment quand il est lourd de processus de répression, stigmatisation, étiquetage... La logique de l'ennemi intérieur est donc le produit et, en même temps, la manifestation la plus aiguë de cette pesanteur de l'ordre politique, dans une tension entre sa marge et son centre. Ici, pour faire référence encore une fois à Michel Foucault, il nous semble que l'enjeu d'une véritable transition est une modification de l'ordre du discours (donc d'une transformation des conditions de production du discours) et non pas seulement une mutation des dispositifs politicojuridiques. La production de la figure de l'ennemi intérieur, sa stigmatisation, sa répression, délimitent les bornes d'un certain ordre du discours politique. La réconciliation, la transition vers un autre ordre politique supposeraient alors, peutêtre, la suppression même de la notion d'ennemi, au profit de la notion libérale d'adversaire ${ }^{64}$. Cette mutation de l'ordre du discours est liée intrinsèquement à la question du pluralisme: celui-ci permet en effet une redéfinition de l'idée d'antagonisme, et de la manière dont elle s'incarne dans une société. De ce point de vue, la différence fondamentale entre les deux cas étudiés ici réside dans le fait que le pluralisme apparaît plus enraciné en Italie, bien qu'il soit, à de nombreux égards, imparfait : cet enracinement laisse penser que le passage d'un schéma à un autre peut se produire plus facilement. Quoi qu'il en soit (nous laissons volontairement ces questions ouvertes), nous voudrions pour conclure attirer l'attention sur un point, qui peut être sujet à discussion: il nous semble, après analyse, que, dans le schéma actantiel qui construit l'ennemi intérieur, l'Etat tient une position dominante, en ce qu'il définit l'ordre du discours dans lequel les luttes autour de la figure de l'ennemi vont prendre place, en ce qu'il en produit les bornes. Ces remarques mènent aussi à remettre en perspective les analyses (par ailleurs parfaitement justifiées) qui mettent en garde contre une "réification » de l'Etat: dans l'ordre du discours, notamment du discours de la répression, la figure de l'Etat est puissante (et cela, au-delà des différences de régimes) et elle prend souvent la forme, non d'une multiplicité d'instances, mais d'une totalité.

\section{NOTES}

1. Sans prétendre définir précisément cette « logique de l'ennemi intérieur »- nous renvoyons sur ce point à la contribution de John Crowley dans le même numéro - nous faisons ici référence à une forme de conflit, souvent à armes inégales, connaissant un 
processus de radicalisation de la stigmatisation mutuelle des parties prenantes, sur un fondement exclusivement politique (et non « ethnique » ou religieux, par exemple) dans les cas qui nous intéressent. Ce processus est bien décrit par Alexandre Koyré : "L'existence de "l'ennemi intérieur" implique et indique la présence au sein de la Cité de groupes non intégrés, non embrassés par le lien social ; de groupes qui se refusent à s'identifier avec le Tout de la Cité, ainsi que de se solidariser - dans ce Tout - avec les autres groupes qui le composent et le constituent; des groupes qui s'isolent - ou se trouvent isolés - dans ce Tout ; qui, l'opposition s'intensifiant et s'exaspérant, passent de l'opposition à l'hostilité, de l'hostilité à la haine ; le cas échéant la lutte sourde se transformera en lutte ouverte : la sédition fera son entrée dans l'Etat ». Alexandre Koyré, La Cinquième colonne, Paris, Allia, 1997 (1945), p. 15. Ce qui peut être ici compris comme un processus volontaire de « sécession » prend surtout la forme d'une fiction construite par l'une au moins des parties adverses, dans les situations que nous étudions ici.

2. Pour reprendre l'expression employée par Vladimir Jankélévitch, Le Pardon, Paris, Aubier, 1967, pp. 21-77.

3. Si l'option de « réconciliation » est souvent définie par les acteurs comme la « solution politique » de sortie du conflit, l'usage de cet expression ne doit pas masquer le fait que la répression est aussi, pleinement, une solution politique à la disposition du pouvoir

4. Nous utilisons délibérément les guillemets pour marquer, d'une part le fait que le terme est employé par les acteurs, d'autre part le fait que sa signification est sujette à caution.

5. Systèmes politiques caractérisés par une instabilité endémique et une " prétorianisation » marquée, en Argentine, ou par une relative stabilité institutionnelle n'ayant pas empêché l'essoufflement du système, notamment partisan, et une polarisation croissante, en Uruguay et au Chili.

6. 164 « disparus » en Uruguay, quelques 1000 au Chili et de 9 à 15000 en Argentine. Sur la disparition-forcée, voir « Disparitions », Cultures \& Conflits, n¹3-14, Paris, L'Harmattan, printemps-été 1994.

7. Il faut d'emblée noter que cette distinction ne saurait être totalement étanche : au contraire, des points de recoupement existent entre l'« aire » de l'autonomie et les organisations militarisées. Toutefois, elles n'ont pas le même type de direction politique, et, surtout, ne poursuivent pas les mêmes objectifs : l'Autonomie ne partage pas, dans sa grande majorité, l'objectif de destruction de l'Etat qui est celui des BR. 8. La première loi Cossiga de 1979 prolonge de six à onze ans le délai de détention préventive autorisé en cas de délits de terrorisme.

9. Non que l'assassinat d'Aldo Moro bouleverse la totalité du système politique mais, outre les effets qu'il entraîne pour les mouvements armés (attraction, recrutement rapide et dissensions), il accélère peut-être l'échec du " compromis historique ", suscite des dissensions au sein de la démocratie chrétienne et provoque une radicalisation de la répression.

10. Pour une justification de l'usage des guillemets, cf. Isabelle Sommier, Le Terrorisme, Paris, Flammarion (Dominos), 2000.

11. Pour évoquer la " criminalité bureaucratique » analysée notamment par Pierre Legendre, Leçons VII, Le Désir politique de Dieu, Etude sur les montages de l'Etat et du droit, Paris, Fayard, 1988, p. 357 sq. 
12. Le 22 décembre 1986, le Parlement uruguayen adopte la loi de « caducité de la prétention punitive de l'Etat » (amnistie dont la constitutionnalité sera confirmée le 2 mai 1988). Le lendemain de l'adoption de la loi, le Grupo de Madres y Familiares de Uruguayos Detenidos-Desaparecidos appelle à un référendum national pour son annulation (conformément à la Constitution de 1967 prévoyant un référendum d'initiative populaire, qui exige les signatures de $25 \%$ du corps électoral). Le référendum, organisé le 16 avril 1989, confirme la loi de « Caducité » (84,7\% des électeurs ont voté, $57 \%$ se sont prononcés en sa faveur)

13. L'étude se concentrera donc principalement sur les discours des instances étatiques, et les procédures mises en œuvre, mais ne vise pas pour autant à réifier la figure de l'Etat, qui fait évidemment l'objet de mutations.

14. Il existe plusieurs lectures des processus de « justice de transition » : la lecture des spécialistes des transitions à la démocratie (J. Colomer, par exemple), qui insiste sur la nécessité d'un compromis (selon le modèle de la théorie des jeux) et sur le caractère " réaliste » de la " réconciliation » décrétée ; celle des juristes internationaux qui fondent leurs analyses sur le caractère obligatoire du droit international prohibant l'amnistie des violations graves des droits de l'homme. On peut aussi mentionner l'idée avancée par Paul Ricoeur du pardon comme « modèle pragmatique » de résolution de conflits, dont l'application politique se veut néanmoins limitée. Cf. Paul Ricoeur, « Le pardon peut-il guérir ?», Esprit, 210, mars-avril 1995, pp. 77-82 et, du même auteur, La Mémoire, l'histoire, l'oubli, Paris, Seuil, 2000, pp. 593-656. Pour plus de précisions sur cette question, cf. Sandrine Lefranc, « La violence d'Etat et le pardon politique ", Raisons politiques, 1, 1998.

15. Le cas de l'Afrique du Sud peut, peut-être, faire figure de contre-exemple. Après le « compromis » qui a permis l'installation d'un gouvernement de transition réunissant le Parti national (parti « historique » de l'apartheid) et le Congrès national africain, puis les élections en 1994, la Constitution provisoire (publiée le 27 avril 1994) prévoyait une loi d'amnistie. La loi de « Promotion de l'unité et de la réconciliation nationale », votée par le Parlement en juillet 1995, optait pour un dispositif beaucoup plus complexe : la Commission de vérité et de réconciliation (qui a présenté une partie de son rapport final au Président Mandela le 29 octobre 1998). Celle-ci était composée de trois comités : le comité sur les violations des droits de l'homme (chargé de faire la "vérité »), le comité sur les réparations et le comité sur l'amnistie. L'amnistie est octroyée sous conditions : le crime doit être politique et être « confessé » de manière exhaustive par le candidat. Si cette confession n'implique pas une demande de pardon, le travail de la Commission est original en ce qu'il a constamment impliqué (au cours des auditions mais aussi dans le recueil des témoignages écrits) les victimes, dans une atmosphère de forte « religiosité ». Par rapport à ses équivalents latino-américains (la CONADEP argentine, 1983 ; la Commission Rettig chilienne de 1990 ; l'Uruguay n'a pas connu de commission de ce type, sinon une commission d'enquête parlementaire peu importante et un rapport publié par l'organisation de défense des droits de l'homme SERPAJ), la commission sud-africaine s'est donnée une mission d'une plus grande ampleur (l'octroi de l'amnistie) et a sans doute représenté un processus de " gestion » des violations passées plus profond, ce qui éloigne l'Afrique du Sud du modèle de la réconciliation unilatéralement décrétée. Alors que les mesures de rémission juridique ont été, dans le cône sud latino-américain, prises par les gouvernements démocratiques (ou, au Chili, validées par le gouvernement Aylwin) à la mise en place d'instances chargées d'établir un panorama historique synthétique et sélectif (pour ce qui est du type de violences 
retenues, les seules « disparitions » et assassinats, le plus souvent), la CVR sud-africaine a produit un panorama historique d'ensemble et fait la vérité sur de nombreux cas individuels de crimes politiques, au moyen des « révélations exhaustives » exigées des candidats à l'amnistie

16. Qui, ne prenant en compte que les «préférences » individuelles, postulent l'indépendance du compromis stratégique de transition par rapport aux représentations du système politique, occultant ainsi la relative continuité de ces dernières.

17. Ce qui correspond à l'un seulement des sens du terme de "réconciliation », qui peut par ailleurs être envisagée comme un recommencement à neuf de la relation, impliquant une révision des identités. Sur la notion d'ordre de discours, cf. Michel Foucault, L'Ordre du discours, Paris, Gallimard, 1972, et La Volonté de savoir, Paris, Gallimard, 1976.

18. Certains mouvements latino-américains, comme les Tupamaros, ont d'ailleurs explicitement influencé les groupes d'extrême gauche italiens.

19. On entend par là, en simplifiant les différences sémantiques entre ces deux termes, la distinction, en partie brouillée par les gouvernements répressifs, entre celui qui a choisi de porter les armes et celui qui s'en tient essentiellement à la parole.

20. Voir notamment Toni Negri, La Dominazione e il sabotaggio, extraits traduits dans Jean-Paul de Gaudemar (dir.), Usines et ouvriers. Le nouvel ordre productif, Rennes, P.U.R., 1998. Plus généralement, sur la thématique opéraïste, voir Mario Tronti, Ouvriers et capital, Paris, C. Bourgois, 1977.

21. Voir sur ce point, Isabelle Sommier, La Violence politique et son deuil. L'après-68 en France et en Italie, Rennes, Presses Universitaires de Rennes, 1998. Voir aussi une typologie des différentes formes d'opéraïsme dans Sergio Bologna, «Qu'est-ce que l'opéraïsme aujourd'hui ? », pp. 48-53 in Marie-Blanche Tahon, André Corten, L'Italie : le philosophe et le gendarme, VLB, Montréal, 1986. Toni Negri lui-même distingue entre différents « axes » de l'autonomie : voir les extraits de son interrogatoire publiés dans Autonomia : post-political politics , NY, Semiotext(e), 1980. Plus généralement, Donatella della Porta, Social movements, II. Violence and the state, Cambridge, Cambridge University Press, 1995 ; Sidney Tarrow, Democrazia e disordine, Beri, Laterza, 1990 ; Michel Wieviorka, Sociétés et terrorisme, Fayard, 1988 ; F. Furet, A. Linier, Terrorisme et démocratie, Fayard, 1985 ; F. Celci, Italie 77, Paris, Seuil, 1977. 22. Cité dans Sergio Marras, Palabra de soldado : Generales Medina Lois, Horacio Toro, Ernesto Baeza y Luis Danus, Santiago, Ediciones del Ornitorrinco, 1989, p. 137. Cf. aussi Alexandra Barahona de Brito, Human Rights and Democratization in Latin America, Uruguay and Chile, Oxford U.P., 1997 ; Jaime Malamud-Goti, Game without end, state terror and the politics of justice, Norman et Londres, University of Oklahoma Press, 1996.

23. On peut se référer ici, à des fins d'illustration, à la distinction ami/ennemi de Carl Schmitt opposée au pluralisme libéral et à la logique de l'adversaire, figure différente de celle de l'ennemi, qui l'accompagne.

24. Cette diversité correspond aussi à l'importance accordée par les analystes à l'idéologie, qui peut être jugée déterminante ou peu significative (le régime autoritaire, contrairement au système totalitaire, pourrait être peu " idéologisé ».)

25. Voir, parmi de nombreuses analyses, les remarques de Dominique Memmi dans «L'engagement politique », in Madeleine Grawitz, Jean Leca (dir.), Traité de science politique, tome 2, Paris, PUF, 1985. 
26. De ce point de vue, les lettres de Moro critiquant l'« insensibilité » de l'Etat et des partis à son sort, et sa « victimisation », sont des documents significatifs.

27. Cf. Paolo Virno (et al.), « Do you remember revolution?», repris in Toni Negri, Italie rouge et noire, Paris, Hachette, 1985.

28. On s'appuie ici sur les travaux d'Isabelle Sommier, De la Criminalisation politique à l'intégration politique, mémoire de DEA, Université de Paris I, 1988 ; et, plus généralement, sur le problème de la répression, Gianfranco Pasquino, La Prova delle armi, Bologne, Il Mulino, 1984 ; Gérard Soulier, « Le terrorisme et l'évolution des droits et des libertés en Europe », in M-B. Tahon, A. Corten, op.cit., et Sergio Zavoli, Le Notte della Republica, Roma, Nuova eri, 1992.

29. Isabelle Sommier, op. cit.

30. Qui permet l'arrestation pour des faits ne relevant pas directement de la violence politique.

31. On verra ultérieurement l'usage de cette notion dans le traitement juridique du « cas Negri »; on y retrouve en tout cas, dans une interprétation « large » du « caractère terroriste », l'esprit du théorème Calogero.

32. Voir, sur l'idée de " grand partage » comme constitutive de l'unité du corps social, Michel Foucault, Histoire de la folie à l'âge classique, Plon, 1962. Cf. aussi plus généralement, sur les processus de construction de l'altérité, Bertrand Badie, Marc Sadoun (dir.), L'Autre. Etudes réunies pour Alfred Grosser, Paris, Presses de SciencesPo, 1998.

33. Il y collabore activement, notamment en menant des enquêtes dans les sections syndicales d'usine, pour dépister les terroristes infiltrés : ici, on observe une autre figure de la logique de l'ennemi intérieur, celle de la contamination.

34. Isabelle Sommier, La Violence politique et son deuil, op. cit.

35. On peut voir ici une manifestation du « mythe du sauveur », qui a aussi été utilisé, à un degré différent, par les militaires en Amérique latine.

36. C'est de ce point de vue que l'on peut comprendre la force légitimante de la référence aux valeurs chrétiennes.

37. Citation du lieutenant-colonel Teófilo Gómez Vera, « La academia superior de seguridad », El Mercurio, 19 décembre 1976.

38. Cf. la distinction opérée par Jean Leca entre " pluralisme politique » et « pluralisme sociétal ». Jean Leca, « La démocratie à l'épreuve des pluralismes », Revue française de science politique, 46(2), avril 1996, pp. 225-279.

39. A l'exemple du Pacte du Club Naval de 1984 en Uruguay, qui définissait les conditions d'une sortie de la dictature, ou de l'accord, au Chili, entre le gouvernement Pinochet et l'opposition réunie au sein de la Concertation démocratique, sur une modification limitée de la Constitution de 1980. Celle-ci interdira aux gouvernements démocratiques d'obtenir une majorité parlementaire suffisante pour l'adoption de lois importantes (notamment en ce qui concerne la " gestion » des violations des droits de l'homme passées) et, a fortiori, de la Constitution (du fait d'un système électoral favorisant les partis de droite et de l'existence de huit sénateurs inamovibles désignés par les institutions du régime militaire).

40. On peut toutefois mentionner un éventuel contre-exemple : en Afrique du Sud, la rhétorique de réconciliation ne s'appuie pas seulement sur une unité et une homogénéité décrétées, mais aussi sur des procédures plurielles, intersubjectives, impliquant une participation des victimes devant la Commission de vérité et réconciliation ou au sein des groupes Khulumani - groupes locaux rassemblant les 
victimes et offrant une médiation par rapport à la CVR. On peut voir dans la tentative sud-africaine une possibilité de faire éclater, de pluraliser l'ordre du discours unitaire, qui préside aux « réconciliations » latino-américaines (on ne préjuge pas ici de son " succès »). D'où le rejet plus ou moins marqué des interprétations historiques de la CVR, tant du côté des acteurs de la lutte contre l'apartheid (divisés dans les pratiques et référents idéologiques), que de celui des tenants de l'ancien régime et de leurs anciens alliés.

41. Cf. sur ce point les rapports d'Amnesty International, de 1980 à 1988, sur la situation judiciaire italienne (disponibles sur Internet).

42. Cf. le documentaire Bella Ciao (1999) sur la situation des émigrés politiques italiens.

43. Une seule loi d'amnistie a été adoptée, en 1946, à l'initiative de Togliatti, au bénéfice de certains fascistes.

44. Voir la rébellion militaire en Argentine, les menaces de Pinochet au Chili.

45. Michel Foucault, La Volonté de savoir, op. cit., pp. 79-80.

46. Le modèle « belliciste » étant fondé sur une confrontation directe et frontale entre deux adversaires, alors que le modèle pastoral s'exerce sur le mode de la direction de conscience. Voir sur ce point Michel Foucault, Il faut défendre la société, Cours au Collège de France 1976, Paris, Seuil, 1998, passim, et La Volonté de savoir, op. cit. 47. Relatif non à l'aune de sa sincérité, mais de son contenu, dans la mesure où il s'agit pour les Eglises catholiques de produire un récit historique pour le moins « nuancé » et pour les représentants des forces armées d'atténuer, sans toutefois rompre radicalement avec elle, l'interprétation des crimes politiques comme des « excès ", rares et imputables aux seuls individus incontrôlables, et le refus d'une responsabilité institutionnelle, les forces armées étant encore souvent considérées comme les « sauveurs » de la patrie. Ces repentances institutionnelles ont surtout pris forme en Argentine, où l'Eglise catholique a été largement associée au régime militaire (quand l'Eglise chilienne a longtemps représenté, au travers du Vicaría de la Solidaridad, la principale opposition " morale » au régime militaire) et où les autorités militaires ont un temps reconnu leur responsabilité dans la violence politique. Le 27 avril 1996, la Conférence des évêques argentins rendait publique la déclaration suivante : «Solidaires de notre peuple et des péchés de tous, nous demandons pardon à Dieu Notre-Seigneur pour les crimes qui ont été commis alors, spécialement pour ceux qui ont eu pour protagonistes les fils de l'Eglise. (...) Nous sommes solidaires de tous ceux qui se sentent blessés par ce drame, et nous regrettons vivement que des fils de l'Eglise aient participé à la violation des droits de l'homme ». Un an auparavant, le général argentin et commandant en chef de l'armée Martín Balza (par la suite répudié par de nombreux officiers) avait déclaré : « Nous ne devons pas nier davantage l'horreur vécue. La fin ne justifiait pas les moyens. L'armée a utilisé des méthodes illégitimes pour obtenir des informations, qui allaient jusqu'à la suppression de la vie. Je ne puis offrir que l'engagement que nous ne répéterons pas les erreurs du passé, dont j'assume toute la responsabilité institutionnelle».

48. Relatif non à l'aune de sa sincérité, mais de son contenu, dans la mesure où il s'agit pour les Eglises catholiques de produire un récit historique pour le moins «nuancé » et pour les représentants des forces armées d'atténuer, sans toutefois rompre radicalement avec elle, l'interprétation des crimes politiques comme des " excès ", rares et imputables aux seuls individus incontrôlables, et le refus d'une responsabilité institutionnelle, les forces armées étant encore souvent considérées comme les « sauveurs » de la patrie. Ces repentances institutionnelles ont surtout pris forme en 
Argentine, où l'Eglise catholique a été largement associée au régime militaire (quand l'Eglise chilienne a longtemps représenté, au travers du Vicaría de la Solidaridad, la principale opposition « morale » au régime militaire) et où les autorités militaires ont un temps reconnu leur responsabilité dans la violence politique. Le 27 avril 1996, la Conférence des évêques argentins rendait publique la déclaration suivante : « Solidaires de notre peuple et des péchés de tous, nous demandons pardon à Dieu Notre-Seigneur pour les crimes qui ont été commis alors, spécialement pour ceux qui ont eu pour protagonistes les fils de l'Eglise. (...) Nous sommes solidaires de tous ceux qui se sentent blessés par ce drame, et nous regrettons vivement que des fils de l'Eglise aient participé à la violation des droits de l'homme ». Un an auparavant, le général argentin et commandant en chef de l'armée Martín Balza (par la suite répudié par de nombreux officiers) avait déclaré : « Nous ne devons pas nier davantage l'horreur vécue. La fin ne justifiait pas les moyens. L'armée a utilisé des méthodes illégitimes pour obtenir des informations, qui allaient jusqu'à la suppression de la vie. Je ne puis offrir que l'engagement que nous ne répéterons pas les erreurs du passé, dont j'assume toute la responsabilité institutionnelle».

49. Reproduits dans Autonomia : post-political politics, op. cit.

50. Intervention du procureur général : «Quand vous parlez sur ce ton surexcité, vous me rappelez (la voix du) coup de fil à Mme Moro » (coup de téléphone passé par les BR durant la détention d'Aldo Moro).

51. Phrase du juge plusieurs fois répétée, après les explications de Negri : «Je n’ai toujours pas compris ce qui vous sépare des BR. ».

52. Théorie de l'interprétation classique dans le discours juridique, mais qui prend un sens particulier dans ce contexte, où l'interprétation textuelle mène à une incrimination.

53. Malgré la décision de la Cour suprême, rendue publique le 8 août 2000, qui rend possible des poursuites judiciaires contre Augusto Pinochet, les poursuites demeurent résiduelles, au sens où l'amnistie reste en vigueur. Les poursuites sont possibles si elles portent sur des faits postérieurs à la date de promulgation de l'amnistie (1978) ou si elles correspondent à un délit jugé « continu » depuis une évolution de la jurisprudence (les « disparitions ») et n'impliquent pas de sanction judiciaire mais le seul établissement de la vérité.

54. L'attribution de la responsabilité se veut individuelle et non collective, la responsabilité n'est pas toujours établie faute d'une enquête judiciaire aboutie, les gouvernements démocratiques insistent sur la dignité et le rôle institutionnel fondamental des forces armées, etc.

55. Pour reprendre l'expression employée par Laura Bonaparte, membre des Madres de Plaza de mayo (Ligne fondatrice).

56. Voir sur ce point les travaux de Didier Bigo, notamment (avec Daniel Hermant), « La relation terroriste ", Etudes polémologiques, 47, mars 1988.

57. Voir la construction de la figure du « grand vieux » (grande vecchio) comme « chef suprême » des organisations terroristes, qui est un des éléments de la focalisation médiatique (négative pour l'essentiel) très forte sur Negri en avril 1979.

58. Position consacrée par les réparations qui ont pu leur être octroyées (même si la plupart des Mères rejettent le principe des réparations matérielles), ainsi qu'à leur « sœurs » chiliennes.

59. Y compris certaines des victimes jusqu'ici perçues comme « radicales ", à l'instar de Pamela Pereira, au Chili, qui a joué un rôle directeur dans le fonctionnement de la Table 
ronde (mesa de diálogo) sur les droits de l'homme, qui a permis récemment l'adoption d'une loi garantissant la confidentialité de l'identité de ceux qui livreraient des informations sur les « disparus».

60. Cf. Paolo Virno, « Do you remember revolution », op. cit., qui présente le repentir comme une continuation de la logique terroriste.

61. Cf. sur ce point la contribution d'Isabelle Sommier, dans le même numéro.

62. Par exemple : «Une pratique sociale terriblement efficace et terriblement ennemie que j'ai subie et qui a contribué à notre défaite : il s'agit d'un événement monstrueux, d'une traduction mystifiée de la violence étatique et de sa vaine fiction de totalité, d'un blitz mystique unilatéral qui nie la libération dans la destruction en lui retirant toute sa dynamique et en ôtant toute la douceur qui existait dans le rapport ", Toni Negri, «Lettre à Félix Guattari », repris in M.-B. Tahon, A. Corten, op. cit.

63. D'autres facteurs, relatifs à l'état du système politique (cf. supra) contribuent évidemment à cette situation.

64. Ces catégories étant utilisées ici sans les jugements de valeur que Schmitt leur attache.

\section{INDEX}

Mots-clés : démocratie, sortie de conflits

Index géographique : Aires géographiques comparées, Amérique du Sud, Italie

Thèmes : Brigades rouges (Italie) 